\title{
Combination of alendronate and genistein synergistically suppresses osteoclastic differentiation of RAW267.4 cells in vitro
}

\author{
MASAYOSHI YAMAGUCHI ${ }^{1}$ and ROBERT M. LEVY $^{2}$ \\ ${ }^{1}$ Department of Hematology and Medical Oncology, Emory University School of Medicine, Atlanta, GA 30322; \\ ${ }^{2}$ Department of Clinical Development, Primus Pharmaceuticals, Inc., Scottsdale, AZ 85253, USA
}

Received November 12, 2016; Accepted March 3, 2017

DOI: 10.3892/etm.2017.4695

\begin{abstract}
Bone is a dynamic tissue that undergoes constant remodeling, with removal by osteoclastic bone resorption and replacement via osteoblastic bone formation and mineralization. Deterioration of bone mass with aging leads to osteoporosis. Bisphosphonates are potent inhibitors of osteoclastic bone resorption. Genistein, an isoflavone, exerts a bone anabolic effect by suppressing osteoclastic bone resorption and stimulating osteoblastic bone formation. The present study was undertaken to investigate the anabolic effects of a combination of alendronate and genistein on osteoclastic differentiation. Preosteoclastic RAW267.4 cells were cultured with alendronate $(0.1-100 \mu \mathrm{M})$ and/or genistein (0.1-100 $\mu \mathrm{M})$ in vitro. Alendronate or genistein alone had no significant effect on the proliferation and death of RAW267.4 cells. Notably, the combination of the two agents was found to potently and synergistically repress the proliferation and death of RAW267.4 cells. Moreover, alendronate or genistein used separately at higher concentrations suppressed the osteoclastic differentiation of RAW267.4 cells induced by receptor activator of nuclear factor- $\mathrm{\kappa B}$ ligand (RANKL) in vitro. However, combinations of the two agents $(0.1-100 \mu \mathrm{M})$ synergistically suppressed the RANKL-induced osteoclastic differentiation. In conclusion, bisphosphonate and genistein combination therapy may provide a novel strategy for the prevention and treatment of osteoclastic bone resorption.
\end{abstract}

\section{Introduction}

Bone homeostasis is regulated through the functions of osteoclasts and osteoblasts (1-3). Bone is a dynamic tissue constantly remodeled by the sequential removal (bone resorption) of

Correspondence to: Dr Masayoshi Yamaguchi, Department of Hematology and Medical Oncology, Emory University School of Medicine, C-5054, 1365C Clifton Road NE, Atlanta, GA 30322, USA

E-mail: yamamasa1155@yahoo.co.jp

Key words: bisphosphonate, alendronate, isoflavone, genistein, osteoclastic differentiation, RAW267.4 cell mature tissue by osteoclasts and its replacement (bone formation) through the deposition of newly formed mineralized matrix by osteoblasts (1-3). Osteoclasts are derived from hematopoietic progenitor cells and osteoblasts develop from bone marrow mesenchymal stem cells $(1,2)$. Bone homeostasis is maintained through the actions of various hormones, cytokines and bone marrow environmental systems (1-3). This disturbance induces bone loss (1). Aging leads to osteoporosis associated with a deterioration of bone mass through suppressed bone formation and promoted bone resorption $(4,5)$. Osteoporosis is widely recognized as a major public health problem $(4,5)$. A notable manifestation of this disease is fracture of the proximal femur, the incidence of which increases as the population ages $(4,5)$. Decreased bone mass in females is primarily due to reduced secretion of estrogen following the beginning of the menopause (5). Osteoporosis is an important cause of morbidity and mortality in elderly women. Development of a new supplemental strategy will be useful in the prevention and treatment of osteoporosis.

Bisphosphonates are a group of drugs that have a structural similarity to pyrophosphate, a high affinity for mineralized tissue, and were developed as agents for inhibiting osteoclastic bone resorption $(6,7)$. These drugs have often been used as the first treatment option for osteoporosis since 1960s, when the first bisphosphonates were developed as drugs for human use $(6,7)$. There are two main classes of bisphosphonates which differ in potency and mode of action, namely the low potency, non-nitrogen-containing bisphosphonates including coronate and ternate, and the more commonly used higher potency, nitrogen-containing bisphosphonates including alendronate, ibandronate and zoledronate $(6,7)$. Bisphosphonates are widely used for their multimodal bone-sparing action to prevent and treat osteoporosis in postmenopausal women, bone pain and hypercalcemia of malignancy $(6,8,9)$. Oral bisphosphonates such as alendronate, risedronate and etidronate are used beneficially to reduce the risk of skeletal fractures in patients with osteoporosis and in metastatic bone cancer (10). However, recent studies have suggested that the inhibitory effects of bisphosphonates on osteoclasts lead to impaired bone remodeling, bisphosphonate-related osteonecrosis of the jaw, gastrointestinal side effects and risk of cancer (11-13).

Botanical isoflavones, including daidzin, daidzein, genistein and genistein, are contained at relatively high concentrations in soybeans $(14,15)$. Daidzin and genistin are hydrolyzed to 
daidzein and genistein, respectively, by $\beta$-glucosidase in the gastrointestinal tract $(14,15)$. Among isoflavones, genistein has been demonstrated to have potent direct anabolic effects on bone metabolism in vitro (14-20), suggesting a role in the prevention of osteoporosis. Therefore, it is hypothesized that genistein may be useful in the prevention and treatment of osteoporosis.

The present study was undertaken to determine whether the bisphosphonate alendronate and the isoflavone genistein synergistically suppress osteoclastic differentiation using preosteoclastic RAW267.4 cells in vitro. The combination of alendronate and genistein was found to exhibit a synergistic suppressive effect on osteoclastic differentiation in vitro. Thus, such combinations may provide a new strategy for the prevention and treatment of osteoporosis with reduced bisphosphonate toxicity.

\section{Materials and methods}

Materials and cells. Dulbecco's Modification of Eagle's Medium (DMEM) with $4.5 \mathrm{~g} / \mathrm{l}$ glucose, L-glutamine, sodium pyruvate and antibiotics [penicillin and streptomycin $(\mathrm{P} / \mathrm{S})$ ] was purchased from Mediatech, Inc. (Corning, Manassas, VA, USA). Fetal bovine serum (FBS) was from Hyclone (GE Healthcare Life Sciences, Logan, UT, USA). Alendronate, genistein, leukocyte acid phosphatase kits for tartrate resistant acid phosphatase (TRAP) staining and all other reagents were purchased from Sigma-Aldrich (Merck KGaA, Darmstadt, Germany) unless otherwise specified. The receptor activator of nuclear factor- $\kappa \mathrm{B}$ ligand (RANKL) was from R\&D Systems, Inc. (Minneapolis, MN, USA). Reagents were dissolved in $100 \%$ ethanol and sterilized distilled water. Mouse monocytic RAW267.4 cells were obtained from the American Type Culture Collection (Rockville, MD, USA) $(21,22)$.

Cell proliferation. RAW267.4 cells (1x105/ml cells/well; $2 \mathrm{ml}$ medium added per well in 24-well plates) were cultured in DMEM containing 10\% FBS and $1 \% \mathrm{P} / \mathrm{S}$ for 3 days in a water-saturated atmosphere containing $5 \% \mathrm{CO}_{2}$ and $95 \%$ air at $37^{\circ} \mathrm{C}$ (23). The cells were then cultured in DMEM containing $10 \% \mathrm{FBS}$ and $1 \% \mathrm{P} / \mathrm{S}$ in the presence or absence of vehicle (ethanol; final concentration $0.1 \%$ ), alendronate $(0.1,1,10$ or $100 \mu \mathrm{M})$, genistein $(0.1,1,10$ or $100 \mu \mathrm{M})$, or alendronate $(0.1$, $1,10$ or $100 \mu \mathrm{M})$ plus genistein $(0.1,1,10$, or $100 \mu \mathrm{M})$. After culture, cells were detached from each culture dish and counted. Following trypsinization of each of culture dish using $0.2 \%$ trpysin plus $0.02 \%$ EDTA in $\mathrm{Ca}^{2+} / \mathrm{Mg}^{2+}$-free PBS for 2 min at $37^{\circ} \mathrm{C}$, cells detached from the dish were collected following centrifugation at $150 \mathrm{x} \mathrm{g}$ and $4^{\circ} \mathrm{C}$ for $5 \mathrm{~min}$ (23). Cells were resuspended in PBS solution and stained with eosin. Cell numbers were counted under a microscope (Olympus MTV-3; Olympus Corporation, Tokyo, Japan) using a hemocytometer plate. For each dish, the average of two counts was calculated. Cell numbers are presented as the number per well.

Cell death. RAW267.4 cells (1x105/ml cells/well; $2 \mathrm{ml}$ medium added per well in 24-well plates) were cultured in DMEM containing $10 \% \mathrm{FBS}$ and $1 \% \mathrm{P} / \mathrm{S}$ for 3 days when confluence was reached (24). The cells were then cultured for an additional 2 days in the presence or absence of alendronate $(0.1,1,10$ or $100 \mu \mathrm{M})$, genistein $(0.1,1,10$ or $100 \mu \mathrm{M})$, or alendronate $(0.1$,
$1,10$ or $100 \mu \mathrm{M})$ plus genistein $(0.1,1,10$ or $100 \mu \mathrm{M})$. After culture, cells were detached from each culture dish and counted as described for the cell proliferation assay.

Osteoclastogenesis assays and TRAP staining. RAW264.7 cells were cultured in 96-well plates in DMEM supplemented with $10 \% \mathrm{FBS}$ and $1 \% \mathrm{P} / \mathrm{S}$ at a density of $1 \times 10^{4}$ cells/well. Cells were cultured for 6 days with RANKL ( $30 \mathrm{ng} / \mathrm{ml})$ pre-incubated for $10 \mathrm{~min}$ with crosslinking anti-poly-histidine antibody (2.5 $\mu \mathrm{g} / \mathrm{ml}$; IC050P; R\&D Systems, Inc., Minneapolis, MN, USA) to induce osteoclast formation $(21,22)$, in the presence or absence of vehicle (ethanol; final concentration $0.1 \%$ ), alendronate $(0.1,1,10$ or $100 \mu \mathrm{M})$, genistein $(0.1,1,10$ or $100 \mu \mathrm{M})$, or alendronate $(0.1,1,10$ or $100 \mu \mathrm{M})$ plus genistein $(0.1,1,10$ or $100 \mu \mathrm{M})$. After 6 days of culture, the cells were fixed and stained for TRAP, a specific marker of the osteoclast phenotype, using a leukocyte acid phosphatase kit. Briefly, cells were washed with PBS, fixed with $10 \%$ neutralized formalin-phosphate $(\mathrm{pH} 7.2)$ for $10 \mathrm{~min}$, dried and stained with a leukocyte acid phosphatase kit (387A; Sigma-Aldrich; Merck KGaA, Darmstadt, Germany) for staining of tartrate resistant acid phosphatase (TRAP) at room temperature for $90 \mathrm{~min}$. TRAP-positive multinucleated cells (MNCs with at least three nuclei) were considered to be osteoclast-like cells, and the cells were counted using light microscopy (Olympus MTV-3). MNC scores are expressed as the mean \pm standard deviation of six cultures with two replicate wells per data set using different dishes and cell preparation.

Statistical analysis. Statistical analysis was performed using GraphPad InStat software (version 3; GraphPad Software, Inc., La Jolla, CA, USA). Multiple comparisons were performed by one-way analysis of variance, followed by a post hoc Tukey's range test for parametric data. $\mathrm{P}<0.05$ was considered to indicate a statistically significant difference.

\section{Results}

Combination of alendronate and genistein reveals a synergistic suppressive effect on the proliferation of RAW267.4 cells. The effects of the bone metabolism regulators alendronate and genistein on the proliferation of RAW267.4 cells in vitro were examined. RAW267.4 cells were cultured for 3 days in the presence or absence of each compound. Culture with alendronate $(0.1,1.0,10$ and $100 \mu \mathrm{M})$ or genistein $(0.1,1.0,10$ and $100 \mu \mathrm{M})$ individually did not have a significant effect on the proliferation of RAW267.4 cells as compared with that of the control $(0.1 \%$ ethanol vehicle; Fig. 1A and B). Next, the effects of various combinations of alendronate and genistein on the proliferation of RAW267.4 cells in vitro were determined. Notably, the combinations of alendronate and genistein with concentrations $(0.1,1.0,10$ and $100 \mu \mathrm{M})$ that did not independently reveal a significant effect on cell proliferation were found to synergistically suppress cell proliferation (Fig. 1C). Thus, the combination of alendronate and genistein was shown to possess a potent and synergistic suppressive effect on RAW267.4 cells in vitro.

Combination of alendronate and genistein synergistically stimulates the death of RAW267.4 cells in vitro. The effects of alendronate and genistein on the death of RAW267.4 cells in vitro were determined. Cells were cultured for 3 days until 

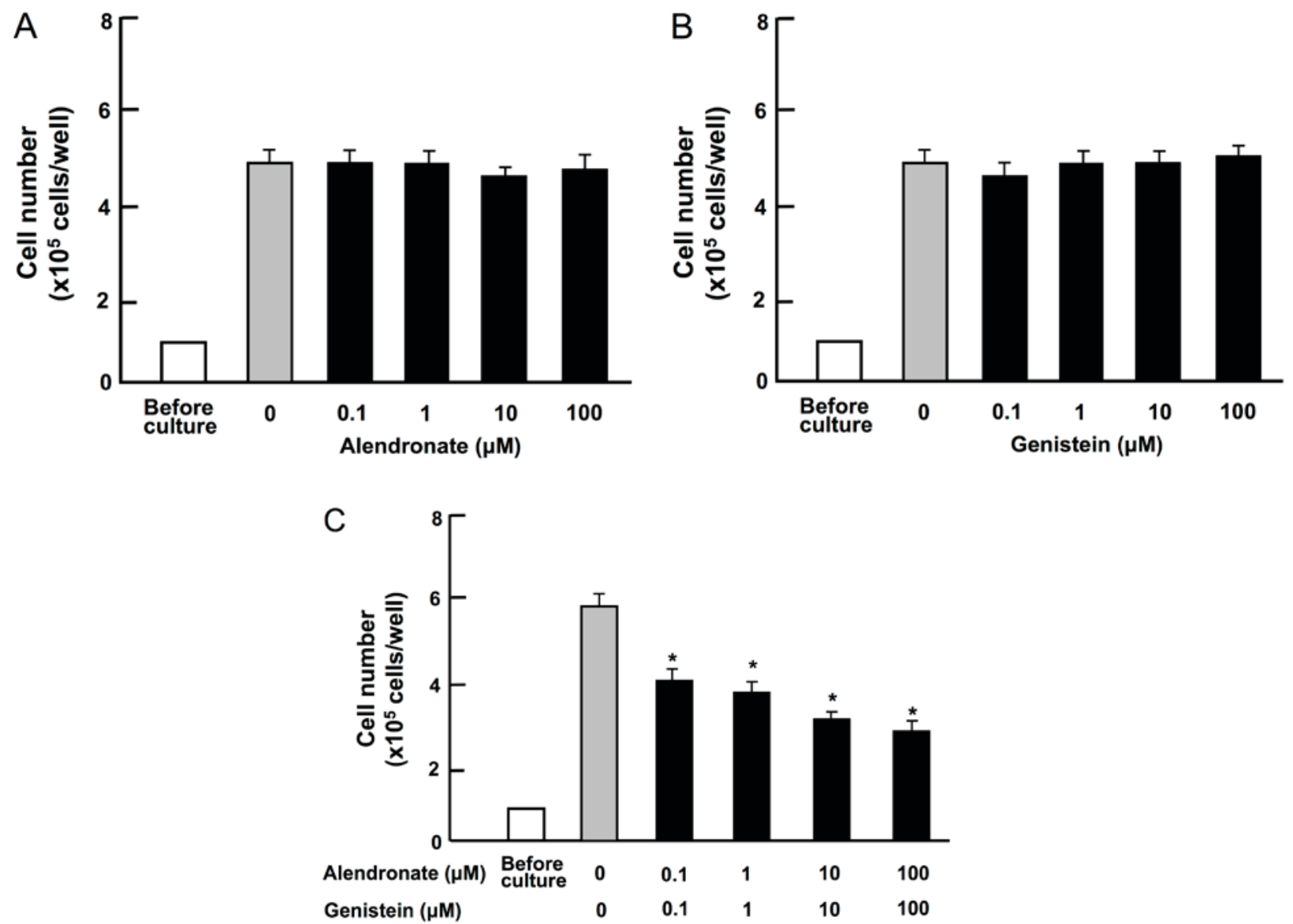

Figure 1. Combination of alendronate and genistein exhibits a synergistic suppressive effect on the proliferation of RAW267.4 cells in vitro. RAW267.4 cells were cultured for 3 days in Dulbecco's modification of Eagle's medium containing vehicle $(0.1 \%$ ethanol), (A) alendronate, (B) genistein or (C) alendronate plus genistein. After culture, the number of attached cells on the dish was counted. Data are presented as the mean \pm standard deviation of two replicate wells per data set using different dishes and cell preparation. " $\mathrm{P}<0.001$ vs. control group (grey bar) as determined by one-way analysis of variance and Tukey-Kramer post hoc test.

they reached confluence, and then the cells were additionally cultured for 2 days in the presence of $0.1 \%$ ethanol, alendronate $(0.1,1.0,10$ and $100 \mu \mathrm{M})$ or genistein $(0.1,1.0,10$ and $100 \mu \mathrm{M})$. Alendronate $(0.1,1.0,10$ and $100 \mu \mathrm{M})$ or genistein $(0.1,1.0,10$ and $100 \mu \mathrm{M}$ ) alone did not cause a significant alteration in the number of RAW267.4 cells (Fig. 2A and B). However, combinations of alendronate $(0.1,1.0,10$ and $100 \mu \mathrm{M})$ and genistein $(0.1,1.0,10$ and $100 \mu \mathrm{M})$ significantly reduced the cell number, indicating that this combination treatment induces cell death (Fig. 2C).

Combination of alendronate and genistein synergistically suppresses osteoclast differentiation of RAW267.4 cells in vitro. To establish an in vitro osteoclastogenesis model suitable for investigation of the activity of combined alendronate and genistein, RAW264.7 monocytic cells were induced to differentiate into osteoclasts by the addition of the key osteoclastogenic cytokine RANKL $(2,3)$. The effects of alendronate and genistein on osteoclast differentiation were tested over a dose range from 0.1 to $100 \mu \mathrm{M}$; the cultures were stained with TRAP 6 days later and osteoclast formation was quantified. RANKL induced robust osteoclast formation (Fig. 3). Culture with alendronate $(0.1,1.0$ and $10 \mu \mathrm{M})$ alone had no significant effect on the RANK-induced enhancement of osteoclastic differentiation
(Fig. 3A). Genistein (0.1 and $1.0 \mu \mathrm{M})$ also did not reveal an effect on RANKL-stimulated osteoclastic differentiation. However, this stimulatory effect of RANKL was suppressed by addition of higher concentrations of alendronate (100 $\mu \mathrm{M}$; Fig. 3A) and genistein (10 and $100 \mu \mathrm{M}$; Fig. 3B). Notably, the combinations of alendronate $(0.1,1.0,10$ and $100 \mu \mathrm{M})$ and genistein $(0.1,1.0$, 10 and $100 \mu \mathrm{M}$ ) were found to exhibit synergistic suppressive effects on the RANKL-induced enhancement of osteoclastogenesis in RAW267.4 cells in vitro (Fig. 3C).

\section{Discussion}

The present study demonstrates the ability of combination of alendronate and genistein to synergistically suppress the proliferation and stimulate the death of preosteoclastic RAW264.7 murine monocytic cells in vitro. Moreover, the combination of alendronate and genistein was found to synergistically suppress the osteoclastic differentiation enhanced by RANKL, a previously demonstrated stimulator of osteoclastic differentiation in RAW264.7 cells $(21,22)$. To the best of our knowledge, this is novel that has not been reported previously, and it may provide a new strategy for the prevention and treatment of bone deterioration induced by osteoclastic bone resorption. 

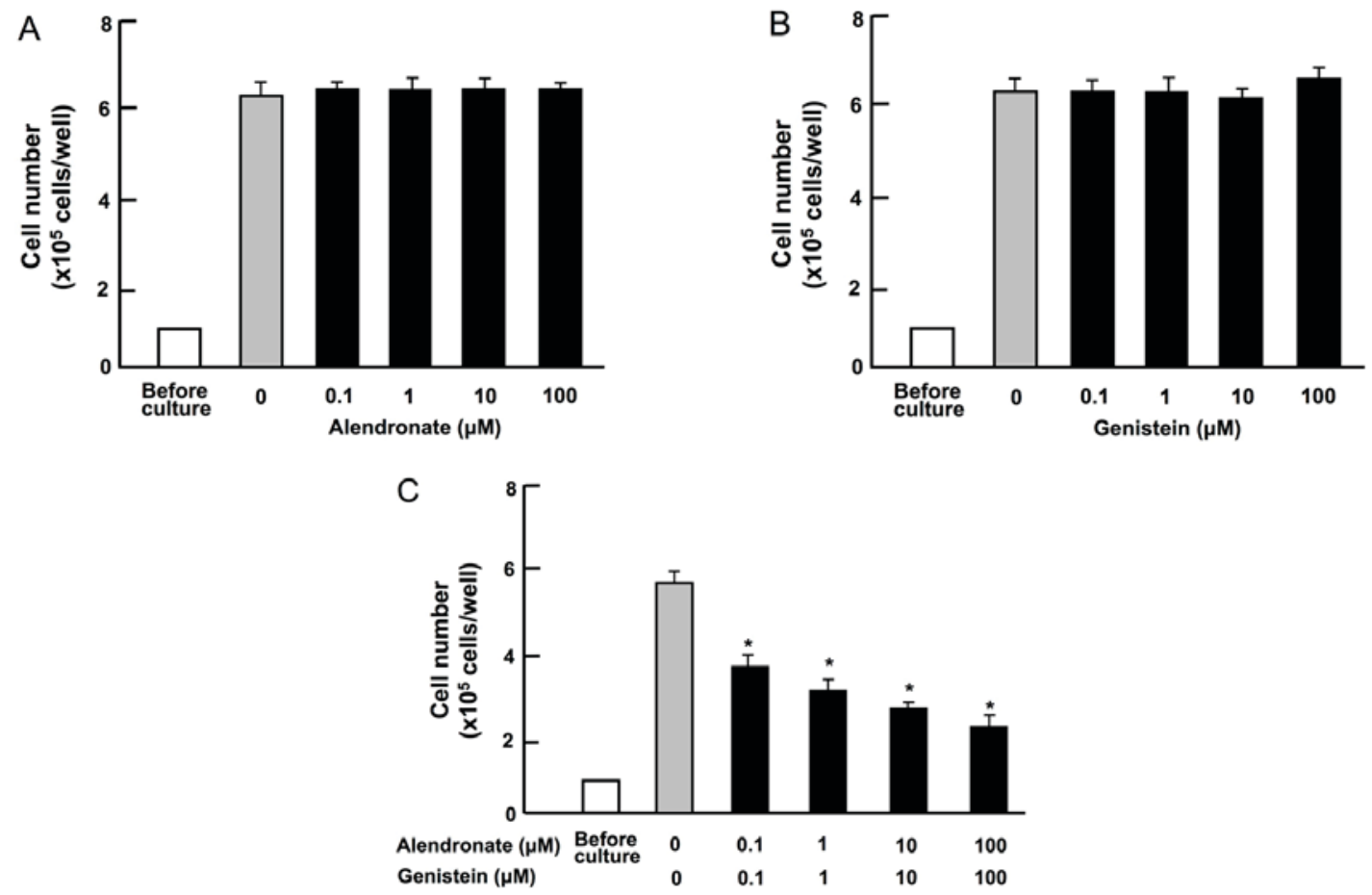

Figure 2. Combination of alendronate and genistein exhibits a synergistic suppressive effect on the death of RAW267.4 cells in vitro. RAW267.4 cells were cultured for 3 days in Dulbecco's modification of Eagle's medium, and then the cells were additionally cultured for 2 days in the presence or absence of (A) alendronate, (B) genistein or (C) alendronate plus genistein. After culture, the number of attached cells on the dish was counted. Data are presented as the mean \pm standard deviation of two replicate wells per data set using different dishes and cell preparation. "P<0.001 vs. control group (grey bar) as determined by one-way analysis of variance and Tukey-Kramer post hoc test.

Alendronate and genistein when used separately did not show significant effects on the proliferation and death of RAW267.4 cells in vitro. Notably, the combination of the two agents acted synergistically to suppress the proliferation and increase the death of RAW267.4 cells, and subsequently suppress the differentiation of preosteoclastic RAW267.4 cells to mature osteoclasts. Furthermore, this combination exhibited a synergistic suppressive effect on RANKL-induced osteoclastic differentiation as compared with the effect of each agent. This suppressive effect on osteoclastic differentiation may be partly based on the proliferation-inhibiting and death-inducing effects of the combination on RAW267.4 cells, which decreased the number of preosteoclastic cells.

Direct effects of genistein on osteoclast precursor differentiation $(25,26)$ and mature osteoclasts $(27)$ have shown that, in addition to stimulating osteoblast function (20), genistein may protect bone by reducing mature osteoclast formation. These effects were observed with genistein concentrations of $>10 \mu \mathrm{M}$ (25-27). Genistein has been shown to have a potent suppressive effect at the later stage of RANKL-induced osteoclastic differentiation in mouse bone marrow cultures (17). Moreover, genistein has been shown to suppress bone resorption in bone tissue culture (16). In the present study, it was demonstrated that genistein negatively regulates the RANKL-induced osteoclastic differentiation of RAW264.7 cells in vitro.

Bisphosphonates have been shown to have a direct suppressive effect on osteoclasts $(6,7,28)$. This effect was observed with an alendronate concentration of $100 \mu \mathrm{M}$, which induced the apoptosis of rabbit osteoclasts in vitro (28). In the present study, combinations of alendronate and genistein with lower concentrations were found to synergistically inhibit the RANKL-induced osteoclastic differentiation of RAW267.4 cells in vitro. These results indicate that this combination may have a potent suppressive effect on osteoclastic bone resorption. Genistein has been indicated to exhibit suppressive effects at the later stage of osteoclastic differentiation and stimulatory effects on the apoptosis of mature osteoclasts (17,25-27), and alendronate may directly stimulate the apoptosis of mature osteoclasts (16). Thus, a combination of alendronate and genistein is speculated to regulate multiple steps associated with osteoclastogenesis and mature osteoclasts. However, additional studies are required to elucidate the mechanism.

There is evidence indicating that bisphosphonate drugs have toxic effects, leading to impaired bone remodeling, bisphosphonate-related osteonecrosis of the jaw, gastrointestinal side effects and risk of cancer (11-13). The present study demonstrated that a combination comprising a relatively low effective dose of a bisphosphonate-type drug and genistein exhibits an inhibitory effect on osteoclastic differentiation. Furthermore, genistein possesses a potent stimulatory effect on osteoblastic bone formation in vitro and in vivo $(15,16)$. The current study findings may provide a new strategy for the prevention and treatment of osteoclastic bone loss in osteoporosis. Combining alendronate with genistein may be useful for reducing the toxicity of the bisphosphonate drug, and may provide potent therapeutic effects. 
A

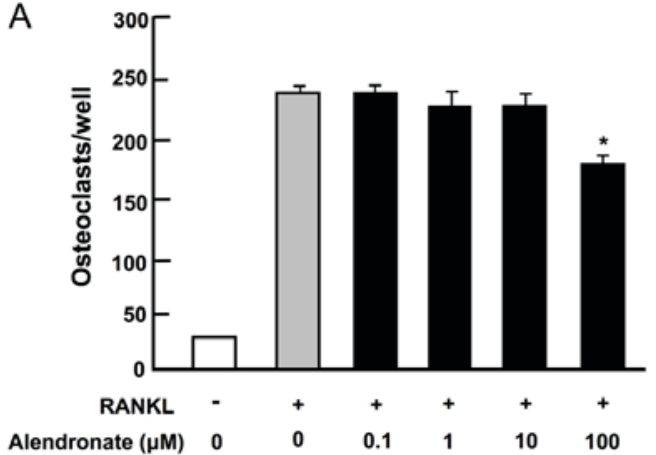

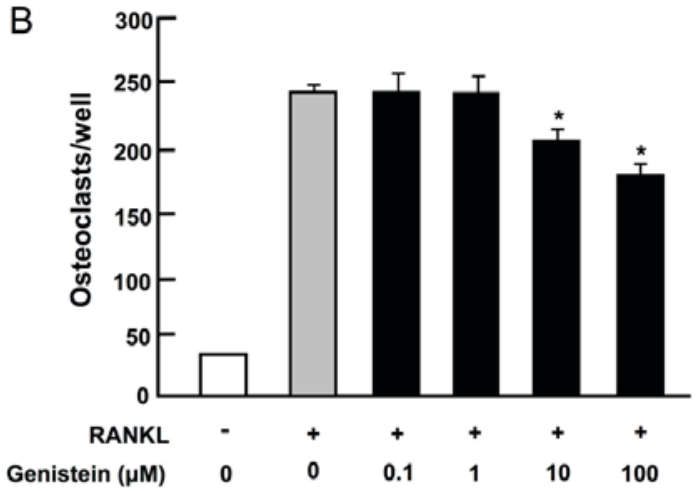

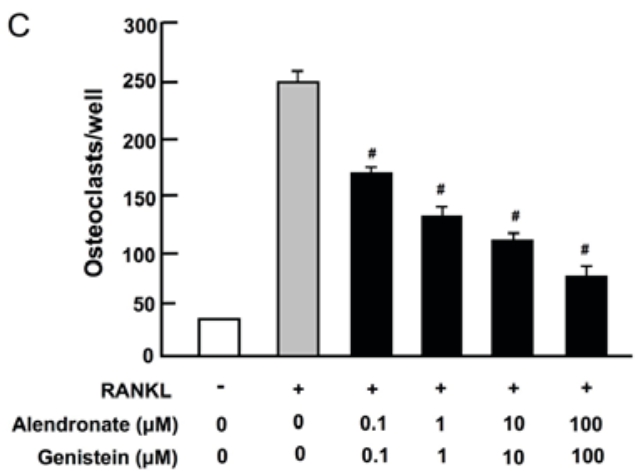

Figure 3. Combination of alendronate and genistein exhibits a synergistic suppressive effect on the osteoclast differentiation of RAW267.4 cells in vitro. Cells were cultured for 6 days with RANKL $(30 \mathrm{ng} / \mathrm{ml})$ pre-incubated for $10 \mathrm{~min}$ with crosslinking anti-poly-histidine antibody ( $2.5 \mu \mathrm{g} / \mathrm{ml})$ in the presence or absence of vehicle $(0.1 \%$ ethanol), (A) alendronate, (B) genistein or (C) alendronate plus genistein. After 6 days of culture, the cells were fixed and stained for TRAP. TRAP ${ }^{+}$multinucleated cells (at least three nuclei) were quantified and averaged from six cultures with two replicate wells per data set using different dishes and cell preparation and are presented as the mean \pm standard deviation. ${ }^{*} \mathrm{P}<0.05$ or ${ }^{\#} \mathrm{P}<0.001$ vs. control group (grey bar) as determined by one-way analysis of variance and Tukey-Kramer post hoc test.

In conclusion, the present study demonstrates that combinations of alendronate and genistein exhibit potent and synergistic suppressive effects on the osteoclastic differentiation of preosteoclastic RAW267.4 cells in vitro. These combinations could potentially inhibit osteoclastic bone resorption. However, further studies are required to investigate the clinical aspects.

\section{References}

1. Raggatt LJ and Partridge NC: Cellular and molecular mechanisms of bone remodeling. J Biol Chem 285: 25103-25108, 2010.

2. Zaidi M, Blair HC, Moonga BS, Abe E and Huang CL: Osteoclastogenesis, bone resorption, and osteoclast-based therapeutics. J Bone Miner Res 18: 599-609, 2003.

3. Chambers TJ and Fuller K: How are osteoclasts induced to resorb bone? Ann N Y Acad Sci 1240: 1-6, 2011.

4. Cooper C and Melton LJ III: Epidemiology of osteoporosis. Trends Endocrinol Metab 3: 224-229, 1995.

5. Weitzmann MN and Pacifici R: Estrogen deficiency and bone loss: An inflammatory tale. J Clin Invest 116: 1186-1194, 2006.

6. Fleisch H: Bisphosphonates: Mechanism of action. Endocr Rev 19: 80-100, 1998 .

7. Mottaghi P: Intravenous bisphosphonates for postmenopausal osteoporosis. J Res Med Sci 15: 175-184, 2010.

8. Caro JJ, Ishak KJ, Huybrechts KF, Raggio G and Naujoks C: The impact of compliance with osteoporosis therapy on fracture rates in actual practice. Osteoporos Int 15: 1003-1008, 2004.

9. Feng Z, Zeng S, Wang Y, Zheng Z and Chen Z: Bishosphonates for the prevention and treatment of osteoporosis in patients with rheumatic diseases: A systematic review and meta-analysis. PLoS One 8: e80890, 2013.

10. Newcomb PA, Trentham-Dietz A and Hampton JM: Bisphosphonates for osteoporosis treatment are associated with reduced breast cancer risk. Br J Cancer 102: 799-802, 2010.
11. Oh YH, Yoon C and Park SM: Bisphosphonate use and gastrointestinal tract cancer risk: Meta-analysis of observational studies. World J Gastroentero 18: 5779-5788, 2012.

12. Faiman B, Pillai AL and Benghiac AG: Bisphosphonate-related osteonecrosis of the Jaw: Historical, ethical, and legal issues associated with prescribing. J Adv Pract Oncol 4: 25-35, 2013.

13. Cardwell CR, Abnet CC, Veal P, Hughes CM, Cantwell MM and Murray LJ: Exposure to oral bisphosphonates and risk of cancer. Int J Cancer 131: E717-E725, 2012.

14. Yamaguchi M and Gao-Balch YH: Role of dietary soybean genistein in osteoporosis prevention. Int J Food Sci Nutr Diet 2: 27-34, 2013.

15. Yamaguchi M: Nutritional factors and bone homeostasis: Synergistic effect with zinc and genistein in osteogenesis. Mol Cell Biochem 366: 201-221, 2012.

16. Yamaguchi M and Gao YH: Inhibitory effect of genistein on bone resorption in tissue culture. Biochem Pharmacol 55: 71-76, 1998.

17. Gao YH and Yamaguchi M: Inhibitory effect of genistein on osteoclast-like cell formation in mouse marrow cultures. Biochem Pharmacol 58: 767-772, 1999.

18. Gao YH and Yamaguchi M: Anabolic effect of daizein on cortical bone in tissue culture: Comparison with genistein effect. Mol Cell Biochem 194: 93-98, 1999.

19. Yamaguchi M and Sugimoto E: Stimulatory effect of genistein and daizein on protein synthesis in osteoblastic MC3T3-E1 cells: Activation of aminoacyl-tRNA synthetase. Mol Cell Biochem 214: 97-102, 2000.

20. Yamaguchi M and Weitzmann MN: The estrogen 17beta-estradiol and phytoestrogen genistein mediate differential effects on osteoblastic NF-kappaB activity. Int J Mol Med 23: 297-301, 2009.

21. Yamaguchi $M$ and Weitzmann $M N$ : The intact strontium ranelate complex stimulates osteoblastogenesis and suppresses osteoclastogenesis by antagonizing NF- $\mathrm{BB}$ activation. Mol Cell Biochem 359: 399-407, 2012.

22. Yamaguchi M and Weitzmann MN: Vitamin K2 stimulates osteoblastogenesis and suppresses osteoclastogenesis by suppressing NF- $\kappa$ B activation. Int J Mol Med 27: 3-14. 2011. 
23. Yamaguchi $\mathrm{M}$ and Daimon $\mathrm{Y}$ : Overexpression of regucalcin suppresses cell proliferation in cloned rat hepatoma H4-II-E cells: Involvement of intracellular signaling factors and cell cycle-related genes. J Cell Biochem 95: 1169-1177, 2005.

24. Izumi T and Yamaguchi M: Overexpression of regucalcin suppresses cell death in cloned rat hepatoma H4-II-E cells induced by tumor necrosis factor-alpha or thapsigargin. J Cell Biochem 92: 296-306, 2004.

25. García Palacios V, Robinson LJ, Borysenko CW, Lehmann T, Kalla SE and Blair HC: Negative regulation of RANKL-induced osteoclastic differentiation in RAW264.7 cells by estrogen and phytoestrogens. J Biol Chem 280: 13720-13727, 2005.
26. Lee SH, Kim JK and Jang HD: Genistein inhibits osteoclastic differentiation of RAW267.4 cells via regulation of ROS production and scavenging. Int J Mol Sci 15: 10605-10621, 2014.

27. Gao YH and Yamaguchi M: Suppressive effect of genistein on rat bone osteoclasts: Involvement of protein kinase inhibition and protein tyrosine phosphatase activation. Int J Mol Med 5: 261-267, 2000.

28. Sutherland KA, Rogers HL, Tosh D and Rogers MJ: RANKL increases the level of Mcl-1 in osteoclasts and reduces bisphosphonate-induced osteoclast apoptosis in vitro. Arthritis Res Ther 11: R58, 2009. 